\title{
WTAP-mediated GPX4 m6A methylation triggers PASMCs ferroptosis and pulmonary vascular fibrosis in pulmonary artery hypertension
}

\author{
Wei Xin ${ }^{1}$, Siyu $\mathrm{He}^{1}$, Yanling $\mathrm{Du}^{2}$, Yang $\mathrm{Yu}^{1}$, Xinyue Song ${ }^{1}$, junting zhang ${ }^{1}$, Yuan Jiang ${ }^{1}$, \\ Songyue $\mathrm{Li}^{1}$, Jingya Zhang ${ }^{1}$, and daling $\mathrm{zhu}^{1}$ \\ ${ }^{1}$ Harbin Medical University \\ ${ }^{2}$ First Affiliated Hospital of Harbin Medical University
}

August 11, 2021

\begin{abstract}
Background and Purpose Ferroptosis is a new form of cell death discovered in recent years. PH is a pulmonary circulatory disease partially characterized by small pulmonary vessel remodeling and fibrosis. However, researchers have not clearly determined whether ferroptosis is involved in $\mathrm{PH}$. Here, this study examined the role and regulatory mechanism of ferroptosis in $\mathrm{PH}$ and pulmonary fibrosis. Experimental Approach To evaluate the occurrence of ferroptosis in rat PH models and in hypoxic PASMCs, MDA, GSH and iron assay were performed. The therapeutic potential of ferroptosis inhibitor fer-1 was evaluated using echocardiography, hemodynamic analysis and ventricular weight measurement in rat PH models. Ferroptosis-related molecule was determined by western blotting and RT-PCR. Changes in autophagy and fibrosis were analyzed by western blotting analysis, RT-PCR and immunofluorescence. Key Results Ferroptosis was existence in vivo and vitro PH models. The fer-1 significantly improved the pathological symptoms of PH and inhibited the occurrence of pulmonary vascular fibrosis. GPX4 was significantly lower expression in PH models, and serves as a key driver of PH-related ferroptosis. A KEGG pathway analysis and RT-PCR detection revealed that GPX4 drives ferroptosis in an autophagy-dependent manner. The RIP experiment verified that WTAP bound to the GPX4 pre-mRNA, induced m6A methylation and promoted its pre-mRNA degradation, thereby reducing the expression of GPX4 in hypoxic PASMCs. Conclusion and Implications This study proposed ferroptosis as a novel form of cell death in $\mathrm{PH}$, and revealed the regulatory mechanism of the ferroptosis in PH, which is based on GPX4 m6A methylation regulated by WTAP.
\end{abstract}

\section{WTAP-mediated GPX4 m6A methylation triggers PASMCsferroptosis andpulmonary vascular fibrosis in pulmonary artery hypertension}

Running title: The ferroptosis regulation mechanism of pulmonary artery hypertension

Wei Xin ${ }^{1,2, \#}$, Siyu $\mathrm{He}^{1,2, \#}$, Yanling $\mathrm{Du}^{3}$, Yang $\mathrm{Yu}^{1,2}$, Xinyue Song ${ }^{1,2}$, Junting Zhang ${ }^{1,2}$, Yuan Jiang ${ }^{1,2}$, Songyue $\mathrm{Li}^{4}$, Jingya Zhang ${ }^{4}$, Daling Zhu ${ }^{1,2,5,6, *}$

1 College of Pharmacy, Harbin Medical University, Harbin 150081, PR China

2 Central Laboratory of Harbin Medical University (Daqing), Daqing 163319, PR China

3 Operating room of the First Affiliated Hospital of Harbin Medical University, Harbin 150001, PR China

4 College of Basic Medicine, Harbin Medical University (Daqing), Daqing 163319, PR China

5 State Province Key Laboratories of Biomedicine-Pharmaceutics of China, Daqing 163319, PR China 
6 Key Laboratory of Cardiovascular Medicine Research, Ministry of Education, Harbin Medical University, Harbin 150081, PR China

\#These authors contributed equally: Wei Xin, Siyu He

*Correspondence to Daling Zhu, College of Pharmacy, Harbin Medical University (Daqing), Xinyang Rd, Daqing, Heilongjiang 163319, P.R. China. E-mail dalingz@yahoo.com

Word counts (8453, excluding figure legends and references)

Abstract (250), Introduction (500), Materials \& Methods (1931), Results (1782), Discussion (1276)

\section{Acknowledgements}

This work was supported by National Natural Science Foundation of China (contract grant Nos. 31820103007,31971057 , and 31771276 to D.Z.);Grant of State Key Laboratory of Respiration Disease (contract grant No. SKLRD-OP-201918 to D.Z.); and Heilongjiang Touyan Innovation Team Program.

\section{Data availability:}

The data that support the findings of this study are available from the corresponding author upon reasonable request. Some data may not be made available because of privacy or ethical restrictions.

\section{Conflict of interest}

None declared.

\section{Author contributions}

WX and SYH conceived and designed experiments and wrote the manuscript and collected and processed data and and participated in the completion of all the experiments. YLD and YY wrote the initial paper and collected and processed data and performed most of the experiment, such as, Western blot analysis, RTqPCR and Immunofluorescence. XYS, JTZ and YJ performed in vitro assays, such as MDA and Glutathione assay. SYL and JYZ performed in vivo assays, such as Immunohistochemistry, Histological and morphological assay. Daling Zhu revised the manuscript and had primary responsibility for final content. All the authors contributed to the interpretation of data and approved the final draft.

\section{Abstract}

\section{Background and Purpose}

Ferroptosis is a new form of cell death discovered in recent years. PH is a pulmonary circulatory disease partially characterized by small pulmonary vessel remodeling and fibrosis. However, researchers have not clearly determined whether ferroptosis is involved in PH. Here, this study examined the role and regulatory mechanism of ferroptosis in $\mathrm{PH}$ and pulmonary fibrosis.

\section{Experimental Approach}

To evaluate the occurrence of ferroptosis in rat PH models and in hypoxic PASMCs, MDA, GSH and iron assay were performed. The therapeutic potential of ferroptosis inhibitor fer- 1 was evaluated using echocardiography, hemodynamic analysis and ventricular weight measurement in rat PH models. Ferroptosisrelated molecule was determined by western blotting and RT-PCR. Changes in autophagy and fibrosis were analyzed by western blotting analysis, RT-PCR and immunofluorescence.

\section{Key Results}

Ferroptosis was existence in vivo and vitro PH models. The fer- 1 significantly improved the pathological symptoms of PH and inhibited the occurrence of pulmonary vascular fibrosis. GPX4 was significantly lower expression in PH models, and serves as a key driver of PH-related ferroptosis. A KEGG pathway analysis and RT-PCR detection revealed that GPX4 drives ferroptosis in an autophagy-dependent manner. The RIP 
experiment verified that WTAP bound to the GPX4 pre-mRNA, induced m6A methylation and promoted its pre-mRNA degradation, thereby reducing the expression of GPX4 in hypoxic PASMCs.

\section{Conclusion and Implications}

This study proposed ferroptosis as a novel form of cell death in $\mathrm{PH}$, and revealed the regulatory mechanism of the ferroptosis in PH, which is based on GPX4 m6A methylation regulated by WTAP.

Keywords : Pulmonary artery hypertension; Ferroptosis; GPX4; fibrosis; autophagy; m6A methylation.

\section{Abbreviations}

GPX4: Glutathione peroxidase 4; GSH: glutathione; fer-1: ferrostatin-1; m6A: N6-methyladenosine; METTL3: methyltransferase-like 3; METTL14: methyltransferase-like 14; PH: Pulmonary artery hypertension; PASMCs: Pulmonary artery smooth muscle cells; WTAP: Wilms' tumor-associated protein.

\section{Introduction}

$\mathrm{PH}$ is a type of pulmonary circulatory disease that is caused by an abnormal increase in pulmonary artery pressure caused by various known or unknown factors, which ultimately leads to right heart failure(D'Alonzo et al., 1991). The main pathological features are spasmodic contractions of the pulmonary artery, intimal hyperplasia and remodeling, and a progressive increase in vascular resistance(Robinson, Pugliese, Fox, \& Badesch, 2016). In this pathological process, the excessive proliferation of PASMCs and resistance to apoptosis are the main causes of abnormal remodeling of the pulmonary artery and PH(Frump et al., 2021). Importantly, excessive PASMCs proliferation promotes the switch of the cells from a static contraction phenotype to a synthetic phenotype, which in turn increases the release of profibrotic factors to drive fibrosis(Samokhin et al., 2018; Zhou et al., 2021). However, the underlying molecular mechanism involved in the regulation of $\mathrm{PH}$ by PASMCs and fibrosis remains unclear.

Ferroptosis is a new form of adjustable programmed cell death that occurs due to the excessive accumulation of iron-dependent lipid reactive oxygen species in the cell(Xie et al., 2016). In contrast to other cell death pathways, such as apoptosis, necrosis and pyroptosis, it has none of the morphological characteristics or phenomena of apoptosis(Mou et al., 2019). It is mainly characterized by the accumulation of lipid reactive oxygen species and iron and the obvious shrinkage of mitochondria with increased membrane density(Dixon et al., 2012). Therefore, the detection of lipid metabolism and iron and glutathione contents are the three effective gold standard indicators to identify ferroptosis. In recent years, an increasing number of studies have found that ferroptosis is closely related to a variety of human diseases. Notably, ferroptosis has been found in many lung diseases, including pulmonary fibrosis, lung cancer and acute lung injury(P. Liu et al., 2020; Rashidipour et al., 2020; Xia, Fan, Zhao, \& Zhu, 2019). However, studies have not determined whether ferroptosis is involved in the process of $\mathrm{PH}$.

The occurrence of ferroptosis is mainly due to the accumulation of lipid peroxides caused by the inactivation of the lipid repair enzyme GPX4, which drives cell death(Ursini \& Maiorino, 2020). Thus, GPX4 may play a crucial role as a key regulatory factor. Intracellular inactivation of GPX4 is mainly due to the inhibition of the cystine/glutamate antiporter system, which leads to the depletion and subsequent inactivation of GSH, which triggers the accumulation of lipid reactive oxygen species (ROS) and finally leads to ferroptosis(Sato et al., 2018). According to reports by Enyong Dai et al., GPX4 may be a prognostic indicator of pancreatic cancer, and the absence of GPX4 might promote the invasion and activation of pancreatic tumors(Dai et al., 2020). Therefore, studies of the regulatory mechanism of GPX4 are the key to solving ferroptosis-related diseases.

In this study, we first observed ferroptosis in PH models in vivo and in vitro. GPX4, the key factor in ferroptosis, was involved in regulating the pathophysiological process of $\mathrm{PH}$ through autophagy-dependent ferroptosis and finally led to the occurrence of pulmonary fibrosis. Moreover, we identified for the first time that WTAP bound to the GPX4 pre-mRNA and caused its degradation, thereby reducing the expression of the GPX4 protein to induce ferroptosis. Our results reveal new mechanisms and signaling pathways related 
to ferroptosis in hypoxic PASMCs, which provide potentially novel targets for the diagnosis and treatment of $\mathrm{PH}$.

\section{Materials and Methods}

\section{Hypoxia-induced PH model}

Adult male Wistar rats with an average body weight of $130 \mathrm{~g}$ were used in this study and were collected from the Experimental Animal Center of Harbin Medical University. All animal experiments were conducted in strict accordance with the guidelines for the management and use of laboratory animals and were approved by the Animal Control and Use Committee (approval number by Animal Committee: HMUDQ20210308001). A controlled environment with an ambient temperature of $22-24^{\circ} \mathrm{C}$, relative humidity of $50 \%$, and a lightdark cycle of $12 \mathrm{~h}$ was provided, as well as food and water to all mice. Wistar rats were randomly divided into three groups: normal, hypoxia, and hypoxia plus ferroptosis inhibitor ferrostatin-1 (fer-1) $(2.5 \mu \mathrm{mol} / \mathrm{kg}$, intraperitoneal injection once daily, for 21 days). The model rats were housed in normal and hypoxic environments for 21 days with a fractional inspired oxygen (FiO2) of 0.21 and 0.12 , respectively. After 3 weeks, rats were anesthetized by pentobarbital $(50 \mathrm{mg} / \mathrm{kg}$, intraperitoneal), and pain was minimized, as described previously(L. Zhang et al., 2020). PH and pulmonary vascular remodeling were assessed, and the lungs were quickly removed.

\section{PASMCs culture}

The lung tissue of adult Wistar rats $(100 \sim 150 \mathrm{~g})$ was extracted. Rats were anaesthetized with

pentobarbital (50 mg/kg, intraperitoneal), and sacrificed by cervical dislocation, as described previously(L. Zhang et al., 2020). The pulmonary artery was carefully peeled off under a microscope, and the tissue mass was digested with $2 \mathrm{mg} / \mathrm{ml}$ collagenase II (Sigma, c6885). After fully digesting the tissue block in a $37^{\circ} \mathrm{C}$ cell incubator, the cell pellet was collected by centrifugation and placed in medium containing $20 \%$ fetal bovine serum and $1 \%$ penicillin and streptomycin. The cells were cultured in a cell incubator at $37^{\circ} \mathrm{C}$ with $5 \% \mathrm{CO} 2$ for 2-3 days to observe the cell morphology and identify smooth muscle cells. Hypoxic cells were cultured in a Tri-GAS incubator and incubated with a gas mixture containing 92\% N2, 5\% CO2, and 3\% O2 for $24 \mathrm{~h}$.

\section{Antibodies and reagents}

The following antibodies and reagents were used: anti-GPX4 (Boster, BM5231), anti-Fibronectin (Boster, BA1772), anti-Collagen-III (Bioss, bs-0549R), anti-WTAP (Santa Cruz Biotechnology, sc-374280), antiMETTL3 (Abcam, ab195352), anti-METTL14 (Bioss, bs-17608R), anti-ALKBH5(Proteintech, 16837-1-AP), anti-FTO (Bioss, bs-7056R), ferrostatin-1 (fer-1)(Selleck, S7243), Erastin, RSL3(Selleck, S8155), Z-VADFMK(Selleck, S7023) and Necrosulfonamide (Selleck, S8251).

\section{siRNA and plasmid construction}

PASMCs were transfected with small interfering RNAs that were designed and synthesized by GenePharma (Shanghai, China) to silence the expression of GPX4, and a nontargeted control siRNA (NC) was used as a negative control. The sequences of siRNAs were as follows: si-GPX4: 5'-CCGAGUGUGGUUUACGAAUTT3', 3'-AUUCGUAAACCACACUCGGTT-5'; si-WTAP-1: 5'-GCGAAGUGUCGAAUGCUUATT3', 3'-UAAGCAUUCGACACUUCGCTT-5'; si-WTAP-2: 5'-GCUGUCCCAGGGCCGUAUUTT3', 3'-AAUACGGCCCUGGGACAGCTT-5'; si-NC: 5'-UUCUUCGAACGUGUCACGUTT-3', 3'TTAAGAGGCUUGCACAGUGCA-5'. The GPX4 (NM_001039849.3) plasmid and control plasmid were designed by GeneChem (Shanghai, China) and verified by sequencing.

\section{Western blot analysis}

The pulmonary arterial tissues of model rats and PASMCs were homogenized and solubilized and extracted with lysis buffer (50 mM Tris, pH 7.4, $150 \mathrm{mM} \mathrm{NaCl}, 1 \%$ Triton X-100, $1 \mathrm{mM}$ EDTA, and $2 \mathrm{mM} \mathrm{PMSF}$ ). The cells or tissues were incubated on ice for 5 minutes, scraped carefully with a scraper, and centrifuged at $13500 \mathrm{rpm}$ for 15 minutes at $4 \mathrm{degC}$, and the supernatant was extracted. Protein extracts $(30-50 \mathrm{~g})$ were 
collected and diluted with a 1:4 ratio of 5xSDS loading buffer: protein extract, mixed with a scroll burner, boiled in a sample boiler at $100 \mathrm{deg} C$ for 5 minutes, and stored at $-80 \operatorname{deg} \mathrm{C}$ until use. The protein samples were fractionated using SDS-PAGE (10\% polyacrylamide gels). The main antibodies against GPX4 (1:200), WTAP (1:200), METTL3 (1:1000), METTL14 (1:1000), ALKBH5 (1:1000), FTO (1:500), BECN1 (1:500), LC3B (1:1000), ATG5 (1:500), Fibronectin (1:1000), Collagen-III $(1,1000)$ and LaminB1 $(1,1000)$ were used, and $\beta$-actin (1:2000) was used as an internal reference protein control.

\section{RT-PCR}

Total RNA was extracted with TRIzol reagent and then reverse transcribed with a Golden 1st cDNA synthesis kit. Finally, the target gene was amplified in a Roche LightCycler 480 (Roche, USA). Primer sequences were as follows: $\beta$-actin rat (sense 5'-CAAGAAGGAAGGCTGGAAAA-3', antisense 5'-AGGGAAATCGTGCGTGAC-3'), GPX4 rat (sense 5'-GAAGCAGGAGCCAGGGAGT-3', antisense 5'ACGCAGCCGTTCTTGTCG3'), GPX4-preRNA rat (sense 5'ACGCAAGTCTGTAGCCCATC-3', antisense 5'-TACCAGTGCCCACATCACAC-3'), PTGS2 rat (sense 5'- CGAGGTGTATGTATGAGTGT-3', antisense 5'-AGTGGGTAAGTATGTAGTGC-3'), FTH1 rat (sense 5'-CGCCAGAACTACCACCAG-3', antisense 5'-TTCAAAGCCACATCATCG-3'), Slc7a11 rat (sense 5'- ATACATTCTGGAGGTCTT-3', antisense 5'- CAGGGTTGTCTACTTCTT-3'), Ascl4 rat (sense 5'- AAGCCAAATCCTTTCTCC-3', antisense 5'- TTCCCATTGTATTCGTCA-3'), BECN1 rat (sense 5'-AATGAATGAGGGCGACAG-3', antisense 5'-TCCATAGGGAACAAGTCG-3'), Atg5 rat (sense 5'-TTGAGGCTCACTTTATGTC-3', antisense 5'-TCACCTGGCTCTTCTTCT-3') and LC3B rat (sense 5'-ACCAAGCCTTCTTCCTCC-3', antisense 5'GCTCTTCTATTTCAAGTCCCTA-3'). The relative expression levels of these mRNAs were analyzed using the $2^{\text {-t }}$ method.

\section{Immunofluorescence}

PASMCs were evenly seeded on glass slides. After transfection or drug administration, the cells were cultured under normoxic or hypoxic conditions for $24 \mathrm{~h}$. Cells were fixed with $4 \%$ paraformaldehyde at $4^{\circ} \mathrm{C}$. The cells were permeabilized with $0.3 \%$ Triton X-100 and blocked with $5 \%$ BSA at room temperature. The primary antibody was incubated with the cells overnight. A FITC-conjugated secondary antibody (1:100) or CY3conjugated secondary antibody $(1: 100)$ was incubated with the cells at $37^{\circ} \mathrm{C}$ in the dark. DAPI (1:200) was used to stain the nuclei. After the anti-fluorescence quenching agent was used to seal the slides, images were captured and analyzed using a living cell workstation. Frozen sections of the model rat lung tissue were obtained, permeabilized with $0.3 \%$ Triton X-100, and the remaining procedures were the same as those described above.

\section{Immunohistochemistry}

Frozen sections of rat lung tissue were removed from the $-80^{\circ} \mathrm{C}$ freezer, equilibrated to room temperature, and sectioned with acetone. The sections were soaked in PBS and sequentially incubated at $37^{\circ} \mathrm{C}$ for 15 minutes with an endogenous peroxidase blocker and blocking serum working solution. Next, the primary antibody was added dropwise and incubated overnight at $4^{\circ} \mathrm{C}$. On the next day, slices were soaked and washed with PBS and sequentially incubated at $37^{\circ} \mathrm{C}$ for 15 minutes with the biotin-labeled goat antimouse/rabbit IgG polymer and horseradish peroxidase-labeled streptomycin working solution. Then, DAB color development, hematoxylin restaining, hydrochloric acid differentiation, rinses with water to return the color to blue, gradient alcohol dehydration, and a xylene treatment for transparency were carried out. Finally, the film was sealed with neutral gum and photographed under a fluorescence microscope.

\section{Histological and morphological analyses}

The lung tissues of rats with pulmonary arterial hypertension were extracted and fixed with $4 \%$ paraformaldehyde for approximately $24 \mathrm{~h}$. The lung tissues were transferred to a $30 \%$ sucrose solution and immersed for approximately 2-3 days. After embedding the lung tissue in embedding solution, frozen sections were obtained using a frozen slicer, and the slices were stored at $-80^{\circ} \mathrm{C}$. These sections were stained with hematoxylin and eosin (HE) or Masson's trichrome stain. The appearance of the tissue was observed using a fluorescence 
microscope (Nikon).

\section{Echocardiography}

A Vevo2100 imaging system (Visual Sonics Inc., Toronto, ON, Canada) and $30 \mathrm{MHz}$ probe were used for echocardiography of the rat model, as previously described24. The pulmonary arterial velocity time integral (PAVTI) was measured.

\section{Hemodynamic analysis and ventricular weight measurement}

Hypoxic model rats were weighed and anesthetized with $3.0 \mathrm{ml}$ of chloral hydrate per $\mathrm{kg}$ body weight. A catheter was inserted into the right ventricle, and the right ventricular pressure was recorded by a computer to evaluate the level of right ventricular pressure. The left ventricle (LV) and interventricular septum were used as the rest of the left ventricle (LV) and weighed with a precision balance. Finally, the $\mathrm{RV} / \mathrm{LV}+$ interventricular septum ratio was used to evaluate the level of right ventricular hypertrophy.

\section{Autophagy flux monitoring}

The mRFP-GFP-LC3 adenovirus was obtained from Hanbio Biotechnology (HB-AP210 0001). PASMCs were transfected with the siRNA or overexpression plasmid, incubated with the mRFP-GFP-LC3 adenovirus, and then incubated with medium containing $5 \%$ serum for $24 \mathrm{~h}$. A living cell workstation was used to capture images and monitor autophagy flux for subsequent analysis.

\section{Nuclear and cytoplasmic protein extraction assay}

The nuclear and cytoplasmic protein extraction kit was purchased from Beyotime (P0028). The experiment was carried out according to the manufacturer's instructions.

\section{MDA assay of lipid peroxidation}

A lipid oxidation (MDA) assay kit purchased from Beyotime (S0131M) was used to detect lipid oxidation levels in PASMCs and lung tissues. Tissues or cells were homogenized to produce lysates. The supernatant was collected, and the protein concentration was determined. A $100 \mu \mathrm{l}$ sample was added to $200 \mu \mathrm{l}$ of MDA detection working solution. After mixing, the mixture was heated at $100^{\circ} \mathrm{C}$ for 15 minutes, cooled to room temperature, and centrifuged at $1000 \mathrm{~g}$ at room temperature for 10 minutes. Two hundred microliters of supernatant were added to a 96-well plate, and then the absorbance was measured at $532 \mathrm{~nm}$ using an enzyme plate reader. The MDA content in the sample was calculated as $\mu \mathrm{mol} / \mathrm{mg}$ protein or $\mu \mathrm{mol} / \mathrm{mg}$ tissue.

\section{GSH assay}

PASMCs and lung tissues were extracted according to the manufacturer's instructions provided with the Glutathione Assay Kit (Sigma, CS0260). Briefly, $10 \mu \mathrm{l}$ of sample were placed into a 96-well plate, and 150 $\mu \mathrm{l}$ of working solution were added to each sample well and mixed well. The mixture was incubated at room temperature for 5 minutes, $50 \mu \mathrm{l}$ of diluted NADPH solution was added and mixed, and the absorbance was determined at $412 \mathrm{~nm}$.

\section{Iron assay}

Lung tissue $(10 \mathrm{mg})$ or PASMCs $(2 \times 106)$ were homogenized in 4-10 volumes of iron assay buffer obtained from Sigma-Aldrich (MAK025, USA). Then, the sample was centrifuged at $16000 \times \mathrm{g}$ for 10 minutes at $4^{\circ} \mathrm{C}$ to remove insoluble material. Afterwards, the sample was brought to a final volume of $100 \mu \mathrm{l}$ with iron analysis buffer. Fifty microliters of each sample were placed in a 96-well plate, and the volume was increased to $100 \mu \mathrm{l}$ per well with assay buffer. Five microliters of iron reducer buffer were added to each sample, mixed well and incubated in the dark at $25^{\circ} \mathrm{C}$ for 30 minutes. Then, $100 \mu \mathrm{l}$ of iron probe were added to each well containing the standard sample and test sample, mixed well and incubated in dark at $25^{\circ} \mathrm{C}$ for 60 minutes. Finally, the absorbance at $593 \mathrm{~nm}$ (A593) was measured to estimate the iron content in the sample.

\section{RNA binding protein immunoprecipitation (RIP)}


After the hypoxia treatment of PASMCs and successful modeling, an RIP kit (Sigma, RIP-12RXN) was used to detect the binding between mRNAs and proteins according to the manufacturer's instructions.

\section{Analysis of mRNA stability}

PASMCs were transfected with WTAP siRNAs or the control siRNA before being exposed to actinomycin $\mathrm{D}(1 \mu \mathrm{g} / \mathrm{ml})$ for $0 \mathrm{~h}, 6 \mathrm{~h}, 12 \mathrm{~h}$ and $24 \mathrm{~h}$. The relative expression of GPX4 was detected using qPCR and normalized to the values measured in the $0 \mathrm{~h}$ group.

\section{Quantification of m6A RNA methylation}

PASMCs were transfected with WTAP siRNAs or the control siRNA. Total RNA was extracted and processed using an RIP kit (Sigma, RIP-12RXN). Total m6A levels in mRNA were measured using an EpiQuik m6A RNA Methylation Quantification Kit (Colorimetric) (Epigentek, NY, USA) according to the manufacturer's instructions.

\section{Bioinformatics software and website}

Metascape, String, GENEMANIA and inBioDiscover prediction websites were used for pathway predictions and the KEGG analysis.

\section{Statistical analysis}

The data were analyzed using GraphPad Prism 8 (GraphPad software, La Jolla, CA, USA). All data are presented as the means \pm SEM. T tests, one-way ANOVA and Tukey's test were used for statistical analyses. When $\mathrm{p}<0.05$, the difference was statistically significant. All experiments were performed in triplicate.

\section{Results}

\section{Hypoxia triggeres ferroptosis and reduces GPX4 expression}

We constructed hypoxic models to detect three key indexes of ferroptosis, lipid peroxidation, GSH (glutathione) depletion and iron accumulation, and to investigate whether ferroptosis is involved in the development of PH. The levels of MDA, GSH and iron in hypoxic PASMCs were detected. Hypoxia significantly increased ferroptosis indexes, including an increased MDA content due to lipid peroxidation (Fig. 1A), GSH depletion (Fig. 1B) and iron accumulation (Fig. 1C), which were further triggered by a hypoxia treatment with the ferroptosis agonists erastin and RSL3. Attractively, only ferrostatin-1 (fer-1, an inhibitor of ferroptosis), but not Z-VAD-FMK (an inhibitor of apoptosis) or Necrosulfonamide (a specific inhibitor of cell necrosis), more significantly abolished MDA production, GSH depletion, and iron accumulation. Using RT-PCR to screen the related genes of ferroptosis, we found significantly lower GPX4 expression (Fig. S1A). Since GPX4 is an important central regulator of ferroptosis, GPX4 was selected as the key object of hypoxia-induced PH for further research. Western blotting (Fig. 1D) and cellular immunofluorescence staining (Figs. 1E and S1C) revealed significantly decreased expression of GPX4 in hypoxic PASMCs compared with that in the control group in a time-dependent manner (Fig. S1B). We used rats to construct a hypoxic PH model and further determine changes in the expression of the GPX4 protein in vivo. GPX4 expression was significantly reduced in the lung tissue of hypoxic rats compared to that in the normal control group, which was reversed by the inhibitor fer-1 (Figs. 1F and S1D), and similar results were obtained using immunohistochemical staining (Fig. 1G). Finally, the localization of GPX4-positive cells was detected. GPX4 was mainly located in the cytoplasm of PASMCs. Hypoxia reduced the expression of GPX4 in the cytoplasm (Fig. 1H). In conclusion, these results suggest that GPX4 is a key regulator of hypoxia-induced ferroptosis and participates in the development of $\mathrm{PH}$.

\section{Ferroptosis is involved in the progression of $\mathrm{PH}$ in vivo}

A 21-day chronic hypoxic PH rat model was constructed and treated with the ferroptosis inhibitor fer-1 (2.5 $\mu \mathrm{mol} / \mathrm{kg}$ ) to further determine whether ferroptosis is directly involved in regulated the development of $\mathrm{PH}$ in vivo. HE staining, RVSP, right ventricular hypertrophy and echocardiography were used to evaluate the role of ferroptosis in hypoxia-induced $\mathrm{PH}$. The inhibitor fer-1 prevented and reversed vascular remodeling 
(Fig. 2B), reduced the mean RVSP (Fig. 2C) and right ventricular hypertrophy (the ratio of RV weight to LV weight plus that of the septum: RV/LV+S)) (Fig. 2D), and increased PAVTI (Fig. 2E) compared with the control group, while the inhibitor did not affect the body weight of the model mice (Fig. 2A). Moreover, Fer-1 also significantly inhibited ferroptosis in vivo (Fig. 2F-H). Overall, the results suggest that ferroptosis is involved in the development of $\mathrm{PH}$ in vivo and that the ferroptosis inhibitor Fer-1 alleviates the progression of $\mathrm{PH}$.

\section{The GPX4-mediated increase in ferroptosis is associated with pulmonary fibrosis}

The development of pulmonary fibrosis is an important endpoint of $\mathrm{PH}$ (Thenappan, Ormiston, Ryan, \& Archer, 2018). The change in the level of fibrosis in the PH model was evaluated to clarify the possible role of fibrosis in ferroptosis-related PH. Masson's trichrome staining showed that the inhibitor fer-1 significantly reduced collagen deposition induced by hypoxia (Fig. 3A). Western blotting, immunohistochemistry and immunofluorescence staining showed that fer-1 decreased the expression of the fibrosis index proteins Fibronectin and Collagen-III compared with the lung tissues from hypoxic model rats (Figs. 3B and S2A-D). In addition, we used a GPX4 siRNA to knock down GPX4 under normoxic conditions or overexpress GPX4 under hypoxic conditions and pretreated PASMCs to determine whether GPX4 downregulation is directly involved in ferroptosis-induced fibrosis. Western blot analyses confirmed that the GPX4 siRNA increased the level of the fibrosis index proteins under normoxic conditions, while the overexpression of GPX4 under hypoxic conditions significantly decreased their expression (Fig. 3C-D). A similar phenomenon was observed in the cellular immunofluorescence staining (Figs. 3E-F and S2E-F). Based on these findings, ferroptosis induced by low GPX4 expression potentially leads to pulmonary fibrosis.

\section{GPX4 regulatedferroptosis is associated with autophagy activation}

Next, we further examined the ferroptosis pathway regulated by GPX4. By performing a GPX4-related pathway analysis (KEGG), we found a correlation between GPX4 and autophagy (Fig. 4A). Previous studies have confirmed that autophagy is involved in the regulation of ferroptosis-related cell death processes(J. Liu et al., 2020). Therefore, we hypothesized that GPX4 may mediate ferroptosis through the autophagy pathway in hypoxic PASMCs. We studied the changes in autophagy after stable transfection of the GPX4 siRNA or GPX4 plasmid to evaluate this hypothesis. Three key autophagy-related proteins and genes were detected using Western blotting and RT-PCR. As expected, GPX4 silencing significantly increased the expression of BECN1 and LC3B in normoxic PASMCs, while the overexpression of GPX4 in hypoxia significantly decreased the expression of BECN1 and LC3B compared with the control group. These treatments did not significantly affect the ATG5 protein level (Fig. 4B and S4A). The results of the cellular immunofluorescence staining for the autophagy proteins BECN1 and LC3B were consistent with the results described above (Figs. S4C). In vivo, the ferroptosis inhibitor fer-1 reduced the expression of the autophagy proteins BECN1 and LC3B (Fig. S4B). Next, we evaluated the autophagy flux in PASMCs treated with the GPX4 siRNA or GPX4 plasmid by transfecting cells with the eGFP-mRFP-LC3 fluorescent plasmid. Compared with the control group, the yellow and red punctate fluorescence of lysosomes and autophagosomes indicating autophagy were significantly increased in the GPX4 siRNA and hypoxia groups, while the overexpression of GPX4 during hypoxia reduced the fluorescent puncta and inhibited autophagy (Fig. 4C). In conclusion, these data supported the hypothesis that autophagy is activated during the process of hypoxic PASMC ferroptosis through a mechanism regulated by GPX4.

\section{GPX4 induces fibrosis through autophagy-dependent ferroptosis}

We used PASMCs stably transfected with the GPX4 siRNA and treated them with the autophagy agonist rapamycin to detect changes in the ferroptosis indicators MDA, GSH, and iron content and to study the relationship between GPX4-related autophagy activation and ferroptosis and fibrosis. The activation of autophagy further increased the accumulation of MDA and iron and reduced the content of GSH, while the ferroptosis inhibitor fer-1 effectively reversed the phenomenon of ferroptosis (Fig. 4D-F). Additionally, Western blot and immunofluorescence assays confirmed that rapamycin further increased the expression of Fibronectin and Collagen-III induced by the GPX4 siRNA. However, the addition of the inhibitor fer-1 
significantly reduced the expression of fibrosis index proteins (Figs. 4G and S4D). Therefore, autophagydependent ferroptosis regulated by GPX4 is essential for fibrosis.

\section{Hypoxia promotes the interaction between GPX4 and methyltransferase WTAP to activate ferroptosis}

The m6A modification of RNA is a key factor affecting the transportation, localization, translation and degradation of RNA and ultimately regulates gene expression(Zhao, Roundtree, \& He, 2017). We first cultured PASMCs transfected with the GPX4 siRNA or GPX4 plasmid under normoxic and hypoxic conditions and screened m6A-related methyltransferases using Western blotting to explore whether low GPX4 expression was regulated by m6A signaling. The expression of the methyltransferases WTAP and METTL3 was increased in hypoxic PASMCs compared with the control group (Fig. 5A). Subsequently, we detected the expression of WTAP and METTL3 in PASMCs at different hypoxia time points and the lung tissues of PH model rats and observed more stable and higher WTAP expression (Fig. 5B-C). In addition, a significant correlation was identified between WTAP and GPX4 in hypoxic PASMCs (Fig. 5D). Western blotting, tissue immunofluorescence and immunohistochemistry analyses of WTAP expression in vivo showed that hypoxia induce high expression of WTAP, while the inhibitor fer-1 reduced its expression in vivo (Figs. 5E and $\mathrm{S} 5 \mathrm{~A}$ and $\mathrm{C}$ ). In the cellular immunofluorescence experiment, the expression of WTAP changed with changes in GPX4 expression. High expression of WTAP was observed in the GPX4 siRNA group cultured under normoxic and hypoxic conditions, while WTAP expression was significantly reduced in the hypoxic GPX4 overexpression group (Figs. 5F and S5B). In addition, WTAP knockdown reversed the low expression of GPX4 caused by hypoxia, further confirming the upstream and downstream relationship between GPX4 and WTAP (Fig. S5D). We also identified the cellular localization of WTAP. Nuclear and cytoplasmic separation experiments showed that hypoxia increased WTAP levels in the nucleus (Fig. 5G).

PASMCs were transfected with the WTAP siRNA to determine whether WTAP was directly involved in ferroptosis-induced fibrosis. After hypoxia, MDA, GSH and iron levels were detected. Compared with the control group, WTAP knockdown alleviated ferroptosis, effectively reduced MDA and iron contents, and increased GSH production (Fig. 5H). Western blot and cellular immunofluorescence staining experiments showed that the expression of the fibrosis index proteins Fibronectin and Collagen-III and the autophagy indicator proteins BECN1 and LC3B were decreased in PASMCs transfected with the WTAP siRNA (Figs S5E and S6A-B). These data confirmed that GPX4 and WTAP interact during hypoxia and jointly promote the fibrosis process through autophagy-dependent ferroptosis.

\section{WTAP reduces the stability of the GPX4 mRNA by interacting with its pre-mRNA to induce m6A methylation}

We investigated whether m6A methylation induced by WTAP accelerated the degradation of the GPX4 mRNA to elucidate the molecular mechanism between GPX4 and WTAP. PASMCs were first transfected with the WTAP siRNA and then treated with actinomycin D at different hypoxia time points to detect changes in GPX4 mRNA stability. RT-PCR results showed that WTAP knockdown increased the stability of the GPX4 mRNA (Fig. 6A). Accumulating evidence suggests a significant relationship between m6A methylation in the nucleus and pre-mRNAs, thus affecting their splicing(Martinez \& Gilbert, 2018). Therefore, the levels of the GPX4 pre-mRNA and mRNA were detected using RT-PCR in hypoxic PASMCs transfected with the WTAP siRNA. Levels of the GPX4 pre-mRNA and mRNA were decreased compared with those in the control group (Fig. 6B). Subsequently, through the RIP analysis, we found that only the GPX4 pre-mRNA was significantly enriched in the WTAP precipitate, while the mRNA was not significantly enriched (Fig. $6 \mathrm{C}$ ), indicating the interaction between WTAP and the GPX4 pre-mRNA. Finally, we detected the level of m6A in PASMCs and found that WTAP knockdown significantly reduced the level of m6A compared with the control (Fig. 6D). These results confirmed that WTAP mediates the m6A modification of the GPX4 pre-mRNA in hypoxic PASMCs.

\section{WTAP mediates GPX4 driven ferroptosis and fibrosis}

A hypoxic PASMC model cotransfected with the WTAP siRNA and GPX4 siRNA was established to ver- 
ify the association between WTAP and autophagy-dependent ferroptosis-induced fibrosis. We found that ferroptosis inhibited by WTAP knockdown was reactivated by the GPX4 siRNA (Fig. 7A-C). In addition, Western blot analysis showed that dual knockout of WTAP and GPX4 restored the decreased levels of autophagy proteins caused by WTAP knockdown (Fig. S6C). Western blot and cellular immunofluorescence experiments confirmed that the dual-knockout of WTAP and GPX4 in hypoxic PASMCs promoted the expression of the fibrotic proteins Fibronectin and Collagen-III (Figs. 7D-E and S6D). Taken together, WTAP is involved in regulating GPX4-mediated ferroptosis and fibrosis in hypoxic PASMCs.

\section{Discussion}

In this study, we innovatively proposed three new ideas. First, ferroptosis was observed in the course of $\mathrm{PH}$ for the first time. Second, the ferroptosis regulator GPX4, a key protein, promoted PH through autophagy-dependent ferroptosis in hypoxic PASMCs and eventually led to fibrosis. Third, hypoxia caused WTAP to bind to the GPX4 pre-mRNA, promoted its pre-mRNA degradation, reduced GPX4 expression, and induced ferroptosis signals. Our findings reveal the role and mechanism by which GPX4 regulates ferroptosis in hypoxic PASMCs and provide a new insight into the prevention and treatment of PH.

Ferroptosis is a form of programmed cell death that depends on the accumulation of iron and lipid reactive oxygen species(Dixon et al., 2012). Numerous studies have proven that ferroptosis is related to the occurrence and development of degenerative diseases, tumors and ischemia reperfusion injury(Li et al., 2019; Xia et al., 2019; Zou et al., 2020), including pulmonary fibrosis, lung cancer and acute lung injury (P. Liu et al., 2020; Rashidipour et al., 2020; Xia et al., 2019). However, the potential role of ferroptosis in regulating $\mathrm{PH}$ remains unknown. In this study, we identified that hypoxia significantly increased ferroptosis indexes, including increases in the MDA content, GSH depletion and iron accumulation, which were further promoted by the ferroptosis agonists erastin and RSL3. Furthermore, fer-1, an inhibitor of ferroptosis, prevented and reversed vascular remodeling and reduced the mean RVSP and right ventricular hypertrophy in our study. More importantly, our data revealed that fer-1 significantly reduced collagen deposition and the expression of Fibronectin and Collagen-III induced by hypoxia, suggesting that ferroptosis may be a key factor triggering fibrosis. Therefore, our data proved that ferroptosis is a novel important form of programmed cell death contributing to $\mathrm{PH}$ and provided a new insight into the study of the pathogenesis of $\mathrm{PH}$.

In addition to ferroptosis, other forms of programmed cell death, such as apoptosis, necrosis and autophagy, are involved in the pathological process of $\mathrm{PH}$ (Rafikov et al., 2019; Ruffenach et al., 2020; X. Zhou et al., 2019). For example, magnesium transporters are involved in the occurrence of PAH by regulating the proliferation, migration and apoptosis of PASMCs(Wang et al., 2021). Our laboratory have also confirmed that BCAT1 could induce cell autophagy in hypoxic PASMCs(Xin et al., 2020). However, the relationship among programmed cell death forms is unclear. Typical phenomena of apoptosis and necrosis have not been observed in ferroptosis(Stockwell et al., 2017), but the autophagy is involved in the occurrence of ferroptosis(Park \& Chung, 2019). In our study, we used Necrosulfonamide, a specific cell necrosis inhibitor, and Z-VAD-FMK, an apoptosis inhibitor, to test whether necrosis and apoptosis are involved in hypoxiainduced PASMC ferroptosis in vitro. Compared with the fer-1 group, Necrosulfonamide and Z-VAD-FMK did not reverse the accumulation of MDA and iron and the depletion of glutathione, suggesting that apoptosis and necrosis are not influenced by hypoxia-induced PASMC ferroptosis. Our results also confirmed that autophagy is involved in the regulation of ferroptosis cell death processes. As shown in our previous study, programmed death ligand 1 induces cell pyroptosis and leads to pulmonary fibrosis in hypoxic PASMCs(M. Zhang et al., 2020), indicating the complexity of the $\mathrm{PH}$ pathological processes. The different programmed cell death pathways may represent different stages or phenotypes, as well as completely different regulatory mechanisms of the pathological changes in $\mathrm{PH}$.

Current research on the mechanism of ferroptosis mainly focuses on three categories, including the excitotoxic effects caused by iron metabolism disorders and abnormal lipid and amino acid metabolism. The three interact with each other to jointly promote the occurrence and development of ferroptosis. In genomics, the glutamic acid/cystine transport system and the downstream protein GPX4 are the main regulators of ferroptosis and main research objects(Forcina \& Dixon, 2019; Yang et al., 2014). GPX4 is a member of a 
class of antioxidant enzymes. As the substrate of glutathione (GSH), GPX4 is able to degrade $\mathrm{H}_{2} \mathrm{O}_{2}$ and lipid ROS into water and the corresponding alcohols, respectively, thus reducing the level of intracellular lipid hydroperoxides, avoiding oxidative damage to cells, and playing a crucial role in cell survival(Ingold et al., 2018). An increasing number of studies have confirmed that GPX4 is the key and central regulator of ferroptosis(Bersuker et al., 2019; Ingold et al., 2018). For example, knockout of GPX4 leads to ferroptosis related cell death and acute renal failure(Friedmann Angeli et al., 2014). Low expression of GPX4 leads to increased lipid peroxidation, which regulates the pathogenesis of pulmonary fibrosis by enhancing transforming growth factor- $\beta$ signaling(Tsubouchi et al., 2019). In our study, GPX4 expression was downregulated in rat $\mathrm{PH}$ models and hypoxic PASMCs. In addition, knockdown of GPX4 in PASMCs increased the expression of fibrosis markers, indicating that GPX4 was involved in the regulation of PASMC ferroptosis and the development of fibrosis caused by hypoxia. We also confirmed that GPX4 regulated ferroptosis and induced fibrosis in an autophagy-dependent manner in hypoxic PASMCs. To the best of our knowledge, this study is the first to report the relationship between GPX4 and autophagy in PH.

The m6A methylation is a common form of epigenetic modification of RNA that potentially regulates the occurrence and progression of diseases by regulating gene expression through RNA translation and degradation(Sun, Wu, \& Ming, 2019). Three types of catalytic enzymes are involved in this biological process, namely, the "writer" methyltransferase, "eraser" demethylase and "reader" methylation reader protein(Z. Liu, Li, Sun, \& Liu, 2018). Among these enzymes, methyltransferase is a complex consisting of METTL3 and METTL14 that forms a heterodimeric catalytic core and regulates the subunit WTAP. The common composition promotes the methylation reaction(J. Liu et al., 2014; Ping et al., 2014). Previous studies on m6A have mainly focused on abnormal embryonic development and tumors(Cui et al., 2017; Mendel et al., 2018). In recent years, an increasing number of researchers have focused on the role of m6A in lung diseases, including pulmonary fibrosis and lung cancer(Han et al., 2020; Y. Shi et al., 2019), and found that m6A plays an extremely important role in the occurrence and development of lung diseases. Hence, studies aiming to explore the role and mechanism of m6A methylation in the regulation of ferroptosis and $\mathrm{PH}$ are very important.

Gene expression is a large and complex process, and splicing of pre-mRNA precursors is one of the key steps in gene expression, which involves the precise splicing and matching of introns and exons of primary transcripts to produce mature mRNA(Lee \& Rio, 2015). A large amount of direct and indirect evidence indicates that the m6A modification is involved in RNA splicing and regulates pre-mRNA splicing(CoveloMolares, Bartosovic, \& Vanacova, 2018; H. Shi, Chai, Jia, \& Fan, 2020; K. Zhou et al., 2019). Tian LAN et al. showed that the m6A methyltransferase KIAA1429 induces m6A methylation of the GATA3 premRNA, leading to the degradation of the GATA3 pre-mRNA and subsequent proliferation and metastasis of hepatocellular carcinoma(Lan et al., 2019). The N6-methyladenosine demethylase FTO targets pre-mRNAs and regulates alternative splicing and 3'-end processing in human HEK293N6 cells(Bartosovic et al., 2017). However, our experimental data interestingly revealed that WTAP mediated m6A modification was the upstream target of GPX4. The m6A methyltransferase WTAP promoted the methylation of the GPX4 premRNA, leading to the degradation of the GPX4 pre-mRNA and thereby reducing the expression of GPX4. The m6A methylation of the GPX4 pre-mRNA was reduced by knocking down WTAP in hypoxic PASMCs. At the same time, the RIP analysis also confirmed the binding between WTAP and the GPX4 pre-mRNA, and upon WTAP knockdown, the enrichment of GPX4 pre-mRNA by the m6A antibody was significantly reduced, which also confirmed the occurrence of the m6A signal.

However, this research still has some limitations that must be further explored and resolved. First, this study confirmed that the WTAP methyltransferase induced the m6A methylation of the GPX4 pre-mRNA in hypoxic PASMCs, thereby degrading the pre-mRNA and resulting in significantly lower expression of GPX4. However, the specific m6A binding site has not yet been elucidated. In addition, we determined the role of ferroptosis in PH model rats and hypoxic PASMCs, but further studies are needed on samples from patients with $\mathrm{PH}$.

In conclusion, this study proved a new mechanism by which WTAP mediated GPX4 methylation to induce 
ferroptosis in PASMCs. GPX4 promoted pulmonary vascular fibrosis and led to PH through autophagydependent ferroptosis. Our results reveal the important role of ferroptosis in the pathological process of fibrosis and $\mathrm{PH}$ and provide a new molecular basis for prevention and treatment research.

\section{References}

Bartosovic, M., Molares, H., Gregorova, P., Hrossova, D., Kudla, G., \& Vanacova, S. (2017). N6methyladenosine demethylase FTO targets pre-mRNAs and regulates alternative splicing and 3'-end processing. Nucleic acids research, 45 (19), 11356-11370. doi:10.1093/nar/gkx778

Bersuker, K., Hendricks, J., Li, Z., Magtanong, L., Ford, B., Tang, P., . . . Olzmann, J. (2019). The CoQ oxidoreductase FSP1 acts parallel to GPX4 to inhibit ferroptosis. Nature, 575 (7784), 688-692. doi:10.1038/s41586-019-1705-2

Covelo-Molares, H., Bartosovic, M., \& Vanacova, S. (2018). RNA methylation in nuclear pre-mRNA processing. Wiley interdisciplinary reviews. RNA, 9 (6), e1489. doi:10.1002/wrna.1489

Cui, Q., Shi, H., Ye, P., Li, L., Qu, Q., Sun, G., . . . Shi, Y. (2017). mA RNA Methylation Regulates the Self-Renewal and Tumorigenesis of Glioblastoma Stem Cells. Cell reports, 18 (11), 2622-2634. doi:10.1016/j.celrep.2017.02.059

D’Alonzo, G., Barst, R., Ayres, S., Bergofsky, E., Brundage, B., Detre, K., . . . Kernis, J. (1991). Survival in patients with primary pulmonary hypertension. Results from a national prospective registry. Annals of internal medicine, 115 (5), 343-349. doi:10.7326/0003-4819-115-5-343

Dai, E., Han, L., Liu, J., Xie, Y., Zeh, H., Kang, R., . . . Tang, D. (2020). Ferroptotic damage promotes pancreatic tumorigenesis through a TMEM173/STING-dependent DNA sensor pathway. Nature communications, 11 (1), 6339. doi:10.1038/s41467-020-20154-8

Dixon, S., Lemberg, K., Lamprecht, M., Skouta, R., Zaitsev, E., Gleason, C., . . . Stockwell, B. (2012). Ferroptosis: an iron-dependent form of nonapoptotic cell death. Cell, 149 (5), 1060-1072. doi:10.1016/j.cell.2012.03.042

Forcina, G., \& Dixon, S. (2019). GPX4 at the Crossroads of Lipid Homeostasis and Ferroptosis. Proteomics, 19 (18), e1800311. doi:10.1002/pmic.201800311

Friedmann Angeli, J., Schneider, M., Proneth, B., Tyurina, Y., Tyurin, V., Hammond, V., . . . Conrad, M. (2014). Inactivation of the ferroptosis regulator Gpx4 triggers acute renal failure in mice.Nature cell biology, 16 (12), 1180-1191. doi:10.1038/ncb3064

Frump, A., Albrecht, M., Yakubov, B., Breuils Bonnet, S., Nadeau, V., Tremblay, E., . . . Lahm, T. (2021). $17 \beta$-estradiol and estrogen receptor- $\alpha$ protect right ventricular function in pulmonary hypertension via BMPR2 and apelin. The Journal of clinical investigation . doi:10.1172/jci129433

Han, B., Chu, C., Su, X., Zhang, N., Zhou, L., Zhang, M., . . . Zhang, R. (2020). N-methyladenosinedependent primary microRNA-126 processing activated PI3K-AKT-mTOR pathway drove the development of pulmonary fibrosis induced by nanoscale carbon black particles in rats.Nanotoxicology, 14 (1), 1-20. doi:10.1080/17435390.2019.1661041

Ingold, I., Berndt, C., Schmitt, S., Doll, S., Poschmann, G., Buday, K., . . . Conrad, M. (2018). Selenium Utilization by GPX4 Is Required to Prevent Hydroperoxide-Induced Ferroptosis. Cell, 172 (3), 409-422.e421. doi:10.1016/j.cell.2017.11.048

Lan, T., Li, H., Zhang, D., Xu, L., Liu, H., Hao, X., . . . Wu, H. (2019). KIAA1429 contributes to liver cancer progression through N6-methyladenosine-dependent post-transcriptional modification of GATA3.Molecular cancer, 18 (1), 186. doi:10.1186/s12943-019-1106-z

Lee, Y., \& Rio, D. (2015). Mechanisms and Regulation of Alternative Pre-mRNA Splicing. Annual review of biochemistry, 84 , 291-323. doi:10.1146/annurev-biochem-060614-034316 
Li, Y., Feng, D., Wang, Z., Zhao, Y., Sun, R., Tian, D., . . . Tian, X. (2019). Ischemia-induced ACSL4 activation contributes to ferroptosis-mediated tissue injury in intestinal ischemia/reperfusion. Cell death and differentiation, 26 (11), 2284-2299. doi:10.1038/s41418-019-0299-4

Liu, J., Kuang, F., Kroemer, G., Klionsky, D., Kang, R., \& Tang, D. (2020). Autophagy-Dependent Ferroptosis: Machinery and Regulation. Cell chemical biology, $2^{77}$ (4), 420-435. doi:10.1016/j.chembiol.2020.02.005

Liu, J., Yue, Y., Han, D., Wang, X., Fu, Y., Zhang, L., . . . He, C. (2014). A METTL3-METTL14 complex mediates mammalian nuclear RNA N6-adenosine methylation. Nature chemical biology, 10 (2), 93-95. doi:10.1038/nchembio.1432

Liu, P., Feng, Y., Li, H., Chen, X., Wang, G., Xu, S., . . . Zhao, L. (2020). Ferrostatin-1 alleviates lipopolysaccharide-induced acute lung injury via inhibiting ferroptosis. Cellular $\mathscr{E}$ molecular biology letters, 25 , 10. doi:10.1186/s11658-020-00205-0

Liu, Z., Li, L., Sun, H., \& Liu, S. (2018). Link Between m6A Modification and Cancers. Frontiers in bioengineering and biotechnology, 6 , 89. doi:10.3389/fbioe.2018.00089

Martinez, N., \& Gilbert, W. (2018). Pre-mRNA modifications and their role in nuclear processing. Quantitative biology (Beijing, China), 6 (3), 210-227. doi:10.1007/s40484-018-0147-4

Mendel, M., Chen, K., Homolka, D., Gos, P., Pandey, R., McCarthy, A., \& Pillai, R. (2018). Methylation of Structured RNA by the mA Writer METTL16 Is Essential for Mouse Embryonic Development. Molecular cell, 71 (6), 986-1000.e1011. doi:10.1016/j.molcel.2018.08.004

Mou, Y., Wang, J., Wu, J., He, D., Zhang, C., Duan, C., \& Li, B. (2019). Ferroptosis, a new form of cell death: opportunities and challenges in cancer. Journal of hematology 85 oncology, 12 (1), 34. doi:10.1186/s13045019-0720-y

Park, E., \& Chung, S. (2019). ROS-mediated autophagy increases intracellular iron levels and ferroptosis by ferritin and transferrin receptor regulation. Cell death 83 disease, 10 (11), 822. doi:10.1038/s41419-0192064-5

Ping, X., Sun, B., Wang, L., Xiao, W., Yang, X., Wang, W., . . . Yang, Y. (2014). Mammalian WTAP is a regulatory subunit of the RNA N6-methyladenosine methyltransferase. Cell research, 24 (2), 177-189. doi:10.1038/cr.2014.3

Rafikov, R., Nair, V., Sinari, S., Babu, H., Sullivan, J., Yuan, J., . . . Rafikova, O. (2019). Gender Difference in Damage-Mediated Signaling Contributes to Pulmonary Arterial Hypertension. Antioxidants $\mathcal{E}$ redox signaling, 31 (13), 917-932. doi:10.1089/ars.2018.7664

Rashidipour, N., Karami-Mohajeri, S., Mandegary, A., Mohammadinejad, R., Wong, A., Mohit, M., . . . Abiri, A. (2020). Where ferroptosis inhibitors and paraquat detoxification mechanisms intersect, exploring possible treatment strategies. Toxicology , 152407. doi:10.1016/j.tox.2020.152407

Robinson, J., Pugliese, S., Fox, D., \& Badesch, D. (2016). Anticoagulation in Pulmonary Arterial Hypertension. Current hypertension reports, 18 (6), 47. doi:10.1007/s11906-016-0657-2

Ruffenach, G., O'Connor, E., Vaillancourt, M., Hong, J., Cao, N., Sarji, S., . . . Eghbali, M. (2020). Oral 15-Hydroxyeicosatetraenoic Acid Induces Pulmonary Hypertension in Mice by Triggering T Cell-Dependent Endothelial Cell Apoptosis. Hypertension (Dallas, Tex. : 1979), 76 (3), 985-996. doi:10.1161/hypertensionaha.120.14697

Samokhin, A., Stephens, T., Wertheim, B., Wang, R., Vargas, S., Yung, L., . . . Maron, B. (2018). COL3A1NEDD9 targets to promote endothelial fibrosis and pulmonary arterial hypertension. Science translational medicine, 10 (445). doi:10.1126/scitranslmed.aap7294

Sato, M., Kusumi, R., Hamashima, S., Kobayashi, S., Sasaki, S., Komiyama, Y., . . . Sato, H. (2018). The ferroptosis inducer erastin irreversibly inhibits system $\mathrm{x}$ - and synergizes with cisplatin to increase cisplatin's 
cytotoxicity in cancer cells. Scientific reports, 8 (1), 968. doi:10.1038/s41598-018-19213-4

Shi, H., Chai, P., Jia, R., \& Fan, X. (2020). Novel insight into the regulatory roles of diverse RNA modifications: Re-defining the bridge between transcription and translation. Molecular cancer, 19 (1), 78. doi:10.1186/s12943-020-01194-6

Shi, Y., Fan, S., Wu, M., Zuo, Z., Li, X., Jiang, L., . . . Chen, Y. (2019). YTHDF1 links hypoxia adaptation and non-small cell lung cancer progression. Nature communications, 10 (1), 4892. doi:10.1038/s41467-01912801-6

Stockwell, B., Friedmann Angeli, J., Bayir, H., Bush, A., Conrad, M., Dixon, S., . . . Zhang, D. (2017). Ferroptosis: A Regulated Cell Death Nexus Linking Metabolism, Redox Biology, and Disease. Cell, 171 (2), 273-285. doi:10.1016/j.cell.2017.09.021

Sun, T., Wu, R., \& Ming, L. (2019). The role of m6A RNA methylation in cancer. Biomedicine E pharmacotherapy = Biomedecine \& pharmacotherapie, 112 , 108613. doi:10.1016/j.biopha.2019.108613

Thenappan, T., Ormiston, M., Ryan, J., \& Archer, S. (2018). Pulmonary arterial hypertension: pathogenesis and clinical management. BMJ (Clinical research ed.), 360 , j5492. doi:10.1136/bmj.j5492

Tsubouchi, K., Araya, J., Yoshida, M., Sakamoto, T., Koumura, T., Minagawa, S., . . . Kuwano, K. (2019). Involvement of GPx4-Regulated Lipid Peroxidation in Idiopathic Pulmonary Fibrosis Pathogenesis. Journal of immunology (Baltimore, Md. : 1950), 203 (8), 2076-2087. doi:10.4049/jimmunol.1801232

Ursini, F., \& Maiorino, M. (2020). Lipid peroxidation and ferroptosis: The role of GSH and GPx4. Free radical biology $\&$ medicine, 152 , 175-185. doi:10.1016/j.freeradbiomed.2020.02.027

Wang, D., Zhu, Z., Lin, D., Zheng, S., Chuang, K., Gui, L., . . . Lin, M. (2021). Magnesium Supplementation Attenuates Pulmonary Hypertension via Regulation of Magnesium Transporters. Hypertension (Dallas, Tex. : 1979), 77 (2), 617-631. doi:10.1161/hypertensionaha.120.14909

Xia, X., Fan, X., Zhao, M., \& Zhu, P. (2019). The Relationship between Ferroptosis and Tumors: A Novel Landscape for Therapeutic Approach.Current gene therapy, 19 (2), $117-124$. doi:10.2174/1566523219666190628152137

Xie, Y., Hou, W., Song, X., Yu, Y., Huang, J., Sun, X., . . . Tang, D. (2016). Ferroptosis: process and function. Cell death and differentiation, 23 (3), 369-379. doi:10.1038/cdd.2015.158

Xin, W., Zhang, M., Yu, Y., Li, S., Ma, C., Zhang, J., . . . Zhu, D. (2020). BCAT1 binds the RNA-binding protein ZNF423 to activate autophagy via the IRE1-XBP-1-RIDD axis in hypoxic PASMCs. Cell death E disease, 11 (9), 764. doi:10.1038/s41419-020-02930-y

Yang, W., SriRamaratnam, R., Welsch, M., Shimada, K., Skouta, R., Viswanathan, V., . . . Stockwell, B. (2014). Regulation of ferroptotic cancer cell death by GPX4. Cell, 156 , $317-331$. doi:10.1016/j.cell.2013.12.010

Zhang, L., Ma, C., Wang, X., Bai, J., He, S., Zhang, J., . . . Zhu, D. (2020). MicroRNA-874-5p regulates autophagy and proliferation in pulmonary artery smooth muscle cells by targeting Sirtuin 3. European journal of pharmacology, 888 , 173485. doi:10.1016/j.ejphar.2020.173485

Zhang, M., Xin, W., Yu, Y., Yang, X., Ma, C., Zhang, H., . . . Zhu, D. (2020). Programmed deathligand 1 triggers PASMCs pyroptosis and pulmonary vascular fibrosis in pulmonary hypertension. Journal of molecular and cellular cardiology, 138 , 23-33. doi:10.1016/j.yjmcc.2019.10.008

Zhao, B., Roundtree, I., \& He, C. (2017). Post-transcriptional gene regulation by mRNA modifications. Nature reviews. Molecular cell biology, 18 (1), 31-42. doi:10.1038/nrm.2016.132

Zhou, J., Li, H., Li, L., Li, Y., Wang, P., Meng, X., \& He, J. (2021). CYLD mediates human pulmonary 
artery smooth muscle cell dysfunction in congenital heart disease-associated pulmonary arterial hypertension. Journal of cellular physiology . doi:10.1002/jcp.30298

Zhou, K., Shi, H., Lyu, R., Wylder, A., Matuszek, Ż., Pan, J., . . . Pan, T. (2019). Regulation of Cotranscriptional Pre-mRNA Splicing by mA through the Low-Complexity Protein hnRNPG. Molecular cell, 76 (1), 70-81.e79. doi:10.1016/j.molcel.2019.07.005

Zhou, X., Liu, Z., Zhu, R., Huang, H., Xu, Q., Xu, H., . . . Liu, J. (2019). NSD2 silencing alleviates pulmonary arterial hypertension by inhibiting trehalose metabolism and autophagy. Clinical science (London, England : 1979), 133 (9), 1085-1096. doi:10.1042/cs20190142

Zou, Y., Henry, W., Ricq, E., Graham, E., Phadnis, V., Maretich, P., . . . Schreiber, S. (2020). Plasticity of ether lipids promotes ferroptosis susceptibility and evasion. Nature, 585 (7826), 603-608. doi:10.1038/s41586-020-2732-8

Figure legends

Figure 1. The expression of GPX4 in hypoxia induced pulmonary arterial hypertension models. (A-C) MDA, GSH and iron levels were assayed in hypoxic PASMCs treated with the agonists erastin and RSL3 or the inhibitors fer-1, Z-VAD-FMK and Necrosulfonamide $(n=6)$. (D) Western blot analysis of GPX4 expression in hypoxic PASMCs $(n=10)$. (E) Immunofluorescence staining was performed to detect the expression of GPX4 in PASMCs. Scale bar $=50 \mu \mathrm{m}(\mathrm{n}=5)$. (F) GPX4 protein levels in pulmonary tissues from hypoxic model rats were determined using Western blot analysis $(n=5)$. (G) Immunohistochemical staining showing GPX4 expression in hypoxic model rats. Scale bar=100 $\mu \mathrm{m}(\mathrm{n}=5)$. (H) GPX4 was mainly localized in the cytoplasm of hypoxic PASMCs, and its expression was decreased $(n=5)$. Statistical analyses were performed with one-way ANOVA or Student's t test. All values are presented as the means \pm SEM. ${ }^{*} \mathrm{p}<0.05 ;{ }^{* *} \mathrm{p}<0.01 ;{ }^{* *} \mathrm{p}<0.001 ;$ and ns, not significant.

Figure 2. The ferroptosis inhibitor fer-1 suppressed the progression of hypoxic PAH in vivo.

Model rats with chronic hypoxic PAH were established and intraperitoneally injected with the inhibitor fer-1 $(2.5 \mu \mathrm{mol} / \mathrm{kg})$. (A) The weight of model rats $(\mathrm{n}=5)$. (B) Morphological changes in the pulmonary artery were examined using HE staining. Scale bar $=100 \mu \mathrm{m}(\mathrm{n}=5)$. (C-D) Statistical analysis of right ventricular systolic pressure (RVSP) and RV/left ventricular (LV) + S weight ratio in each model rat group (n=5). (E) The pulmonary artery velocity time integral (PAVTI) was measured using echocardiography to evaluate the pulmonary arteries in different groups $(\mathrm{n}=5)$. (F-H) MDA, GSH, and iron levels were assayed in the lung tissues from model rats $(\mathrm{n}=5)$. Statistical analyses were performed with one-way ANOVA. All values are presented as the means \pm SEM. ${ }^{*} \mathrm{p}<0.05 ;{ }^{* *} \mathrm{p}<0.01 ;$ and ${ }^{* * *} \mathrm{p}<0.001$.

Figure 3. Pulmonary fibrosis was regulated by GPX4-mediated ferroptosis. (A) Pulmonary fibrosis in rats treated with the inhibitor fer-1 was observed using Masson's trichrome staining. Scale bar $=100 \mu \mathrm{m}$ $(\mathrm{n}=5)$. (B) Western blot analysis of Fibronectin and Collagen-III expression in the pulmonary tissues from hypoxic model rats treated with fer-1 $(\mathrm{n}=5)$. (C-D) Western blot analysis of Fibronectin and CollagenIII protein expression in PASMCs transfected with the GPX4 siRNA or GPX4 plasmid (n=8). (E-F) Immunofluorescence staining for Fibronectin and Collagen-III in PASMCs treated with the GPX4 siRNA or GPX4 plasmid. Fibronectin and Collagen-III (red), and DAPI (blue). Scale bar $=100 \mu \mathrm{m}(\mathrm{n}=5)$. Statistical analyses were performed with one-way ANOVA. All values are presented as the means \pm SEM. ${ }^{*} \mathrm{p}<0.05$; ${ }^{* *} \mathrm{p}<0.01 ;{ }^{* * *} \mathrm{p}<0.001 ;$ and ns, not significant.

Figure 4. GPX4 induced fibrosis through autophagy-dependent ferroptosis. (A) Prediction of GPX4 related pathways. (B) Western blot analysis of the expression of the autophagy indicator proteins BECN1, LC3B and Atg5 in PASMCs transfected with the GPX4 siRNA or GPX4 plasmid (n=7). (C) Determination of autophagy flux in PASMCs cotransfected with the GPX4 siRNA or GPX4 plasmid and the eGFP-mRFP-LC3 plasmid and exposed to normoxia or hypoxia for $24 \mathrm{~h}$. Yellow and red dots indicate autolysosomes and autophagosomes, respectively. Scale bar $=50 \mu \mathrm{m}(\mathrm{n}=5)$. (D-F) The levels of MDA, GSH, and iron were assayed $(n=5)$. (G) Western blot analysis of Fibronectin and Collagen-III protein expression 
in PASMCs treated with autophagy agonists and ferroptosis inhibitors $(n=7)$. Statistical analyses were performed with one-way ANOVA. All values are presented as the means \pm SEM. ${ }^{*} \mathrm{p}<0.05 ;{ }^{*} \mathrm{p}<0.01$; $* * * \mathrm{p}<0.001$; and ns, not significant.

Figure 5. The interaction between GPX4 and WTAP was promoted during the ferroptosis of hypoxic PASMCs. (A) Western blot analysis of levels of the m6A-associated proteins WTAP, METTL3, METTL14, ALKBH5 and FTO in PASMCs transfected with the GPX4 siRNA or GPX4 plasmid (n=8). (B) RT-PCR analysis of WTAP and METTL3 expression levels in PASMCs after 0, 6, 12, 24, 48, and 72 $\mathrm{h}$ of hypoxia treatment $(\mathrm{n}=6)$. (C) RT-PCR analysis of the mRNA levels of WTAP and METTL3 in the lung tissues of hypoxic model rats, with rat $\beta$-actin serving as the standard $(n=6)$. (D) Correlation analysis of GPX4 and WTAP expression in hypoxic PASMCs $(n=10)$. (E) Western blot analysis of WTAP and METTL3 expression in lung tissues from hypoxic model rats $(n=5)$. (F) Immunofluorescence staining for WTAP in PASMCs transfected with the GPX4 siRNA or GPX4 plasmid. WTAP (red) and DAPI (blue). Scale bar $=100 \mu \mathrm{m}(\mathrm{n}=5)$. (G) WTAP was mainly localized in the nucleus of hypoxic PASMCs, and its expression was increased $(n=5)$. $(\mathrm{H})$ The levels of MDA, GSH, and iron were assayed $(n=5)$. Statistical analyses were performed with one-way ANOVA or Student's t test. All values are presented as the means \pm SEM. ${ }^{* *} \mathrm{p}<0.01 ;{ }^{* * *} \mathrm{p}<0.001 ;$ ns, not significant.

Figure 6. WTAP reduced the stability of the GPX4 mRNA by interacting with the pre-mRNA to induce m6A methylation. (A) PASMCs were transfected with the WTAP siRNA and treated with actinomycin D to detect the stability of the GPX4 mRNA after 0,6 , and $12 \mathrm{~h}$ of hypoxia treatment $(\mathrm{n}=5)$. (B) RT-PCR analysis of GPX4 pre-mRNA and mRNA levels in PASMCs transfected with the WTAP siRNA; rat $\beta$-actin served as the standard $(n=6)$. (C) The correlations between the levels of WTAP and the GPX4 pre-mRNA or mRNA were detected using RT-PCR after RNA immunoprecipitation (RIP) (n=6). (D) RIP of the GPX4 pre-mRNA and m6A antibody in PASMCs transfected with the WTAP siRNA $(\mathrm{n}=6)$. Statistical analyses were performed with one-way ANOVA and two-way ANOVA. All values are presented as the means \pm SEM. ${ }^{* *} \mathrm{p}<0.01 ;{ }^{* * *} \mathrm{p}<0.001$; and ns, not significant.

Figure 7. WTAP mediated GPX4-driven ferroptosis and fibrosis. PASMCs were cotransfected with the WTAP siRNA and GPX4 siRNA. (A-C) The levels of MDA, GSH, and iron were assayed $(n=5$ or $n=6)$. (D) Western blot analysis of Fibronectin and Collagen-III protein expression in processed PASMCs $(\mathrm{n}=6)$.

(F) Detection of Fibronectin and Collagen-III in PASMCs using immunofluorescence staining. Fibronectin and Collagen-III (red), and DAPI (blue). Scale bar $=100 \mu \mathrm{m}(\mathrm{n}=5)$. Statistical analyses were performed with one-way ANOVA. All values are presented as the means \pm SEM. ${ }^{* *} \mathrm{p}<0.01$ and ${ }^{* * *} \mathrm{p}<0.001$.

\section{Hosted file}

1.pdf available at https://authorea.com/users/430242/articles/533768-wtap-mediated-gpx4-m6amethylation-triggers-pasmcs-ferroptosis-and-pulmonary-vascular-fibrosis-in-pulmonaryartery-hypertension

\section{Hosted file}

2.pdf available at https://authorea.com/users/430242/articles/533768-wtap-mediated-gpx4-m6amethylation-triggers-pasmcs-ferroptosis-and-pulmonary-vascular-fibrosis-in-pulmonaryartery-hypertension

\section{Hosted file}

3.pdf available at https://authorea.com/users/430242/articles/533768-wtap-mediated-gpx4-m6amethylation-triggers-pasmcs-ferroptosis-and-pulmonary-vascular-fibrosis-in-pulmonaryartery-hypertension

\section{Hosted file}

4.pdf available at https://authorea.com/users/430242/articles/533768-wtap-mediated-gpx4-m6amethylation-triggers-pasmcs-ferroptosis-and-pulmonary-vascular-fibrosis-in-pulmonary- 
artery-hypertension

\section{Hosted file}

5.pdf available at https://authorea.com/users/430242/articles/533768-wtap-mediated-gpx4-m6amethylation-triggers-pasmcs-ferroptosis-and-pulmonary-vascular-fibrosis-in-pulmonaryartery-hypertension
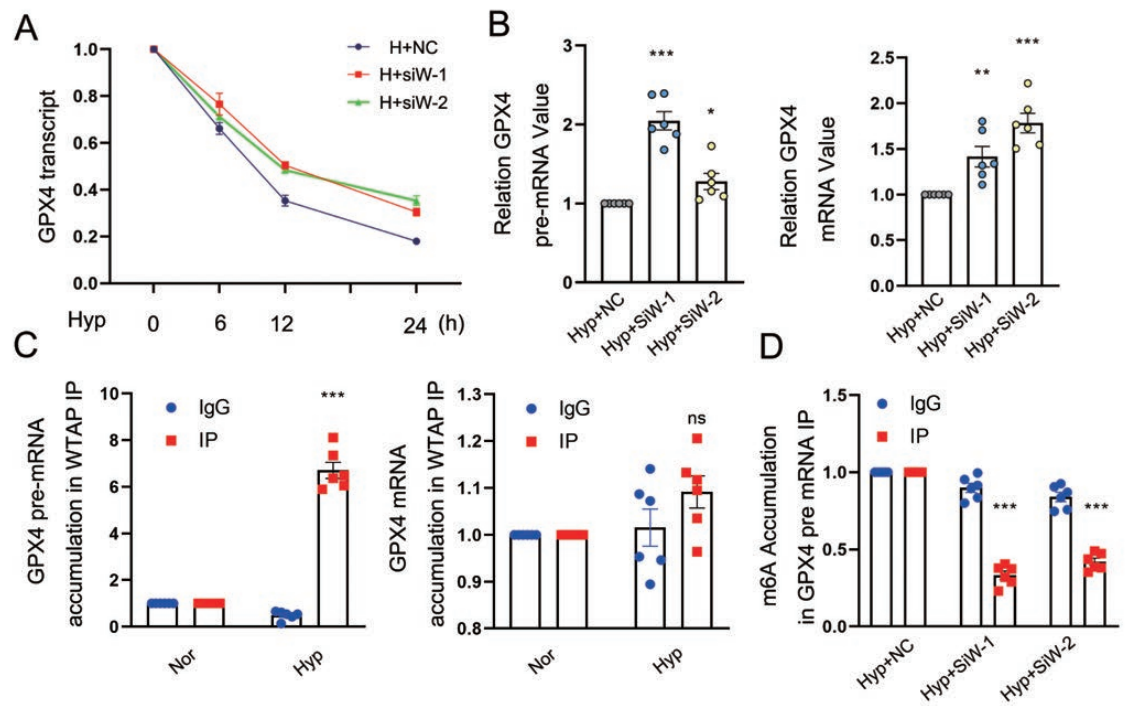

\section{Hosted file}

7.pdf available at https://authorea.com/users/430242/articles/533768-wtap-mediated-gpx4-m6amethylation-triggers-pasmcs-ferroptosis-and-pulmonary-vascular-fibrosis-in-pulmonaryartery-hypertension 